\title{
Téoros
}

Revue de recherche en tourisme

\section{Résidences secondaires et développement régional}

\section{Odette Paré et Marcel Samson}

Volume 5, numéro 1, mars 1986

Tourisme et régions

URI : https://id.erudit.org/iderudit/1080590ar

DOI : https://doi.org/10.7202/1080590ar

Aller au sommaire du numéro

Éditeur(s)

Université du Québec à Montréal

ISSN

0712-8657 (imprimé)

1923-2705 (numérique)

Découvrir la revue

Citer cet article

Paré, O. \& Samson, M. (1986). Résidences secondaires et développement régional. Téoros, 5(1), 24-25. https://doi.org/10.7202/1080590ar d'utilisation que vous pouvez consulter en ligne.

https://apropos.erudit.org/fr/usagers/politique-dutilisation/ 


\title{
Résidences secondaires et développement régional
}

\author{
par Odette Paré et Marcel Samson*
}

Dans certaines municipalités des régions rurales voisines de la région métropolitaine de Montréal, le nombre de résidences secondaires dépasse de beaucoup celui des résidences principales. Ainsi en est-il de plusieurs municipalités localisées dans les MRC des Laurentides, des Pays d'en Haut et de la Matawinie, par exemple. En d'autres endroits, si le rapport n'a pas atteint la méme ampleur, l'implantation de la résidence secondaire a tout de même transformé le paysage.

En fait, la résidence secondaire sous toutes ses formes - cabanon, chalet saisonnier ou habitable à l'année - a connu un développement rapide après la seconde guerre mondiale. Dés la fin des années 1930 cependant, l'intervention du gouvernement québécois ne fut pas étrangère à la main-mise des citadins sur les rives des lacs et des rivières les plus immédiatement accessibles. Plus encore, cette intervention a sans doute été déterminante dans la distribution spatiale de groupes sociaux distinctifs selon les plans $\mathrm{d}^{+}$eau.

\section{Quelques éléments d'histoire}

A partir de la deuxième moitié du $19 \mathrm{e}$ siècle, quelques riches bourgeois, majoritairement anglophones, érigèrent de grandes villas dans la région LaurentidesLanaudière; faute d"intervenants, il $n^{4} y$ avait à cette époque aucun véritable enjeu sur l'espace rural. A cause d'une terre difficile, l'agriculture n'avait jamais wéritablement démarré au-delà de la plaine; seules les compagnies forestières y traçaient les premières voies de pénétration.

Mais I'histoire devait se répéter. Tout "comme dans l'ouest de l'ile de Montréal où l'arrivéte du chemin de fer attira le tourisme d'été, ce sont aussi les chemins de fer, atteignant Saint-Jérômé en 1876, qui ont permis aux premiers flux de touristes de se déverser dans ce futur *terrain de jeux- des Montréalais. Le chroniqueur Arthur Buies raconte qu'en 1891 ndes trains de plaisir amenaient, presque chaque dimanche de la

Marcel Samson, prolesseur-chercheur ef Qdette Paré. assistante de recherche, sont attaches il I'INRS. Urbanisation. Letur texte s' integrera a une btude sur hes rtsidences secondaires subventionné par le Conscil de racherches en sciences humaines du Camada. belle saison, une affuence considerable de nombreut employés de Montreal avides de jouir.. de l'armosphère el des sites pirtoresques. " Ainsi, à cóté des villas luxueuses, de véritables ghettos de villégiateurs saisonniers ont commencé à se constituer: les juifs, les anglophones, les francophones s'aggloméraient pendant la belle saison autour de lacs qui prenaient la *couleur sociale et ethnique: en quelque sorte de ces nouveaux envahisseurs.

\section{Un exemple:}

Saint-Donat, P.Q.

Le chemin de fer s'arrêtant à Rawdon (1910), la région de Saint-Donat fut un peu plus lente à $s^{\dagger}$ ouvrir au tourisme d'hiver mais, à partir de la première guerre, elle illustre bien le developpement qu'a connu l'ensemble du territoire. Dans ce coin de pays, la tentative de colonisation fut relativement brève el déjà, au tournant du sıècle, la plupart des colons avajent abandonné l'agriculture, sauf comme substance familiale d'appoint, pour se tourner vers l'industrie forestière qui s'implantait solidement.

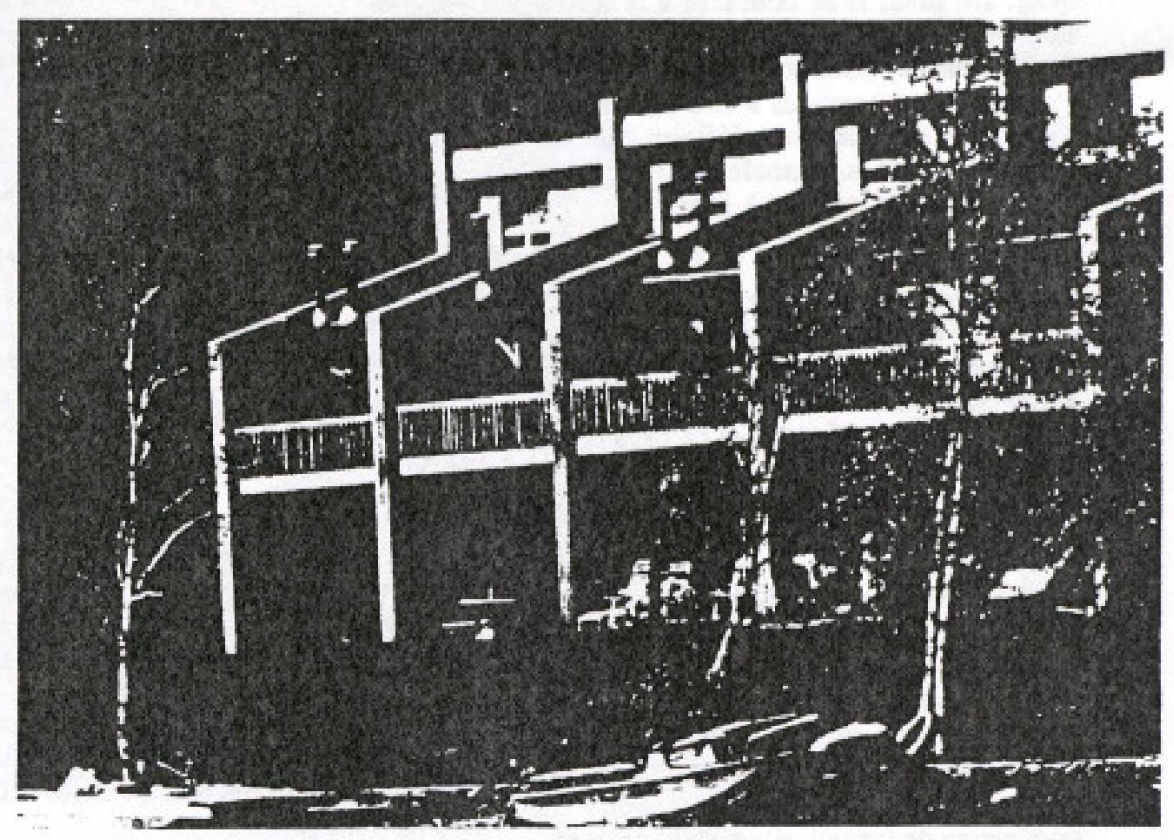

La rfsidence secondaire sous toutes ses formes a connu un développement rapide après la deuxidme guerte mondiale.

Au mème moment, le tourisme commençait à bénéficier de l'apparition de l'automobile, moyen beaucoup plus souple que le chemin de fer. En 1915, une dizaine de vacanciers d'été étaient installés sur les rives du lac Archambault et en bordure du lac Ouareau. Quelque vingt ans plus tard, au cours des annees 1930. Raoul Blanchard affirmait que -Saint-Donat, embelli par les Lacs Ouareau et Archambault, a 8 hôtels, 15 pensions, des camps, plus de 1000 personnes installees pour 6 semaines, ef des visiteurs plus pressés.s. ${ }^{[21}$ A cette époque, le ministère des Terres et Forêts (MTF) du Québec était responsable de la gestion des grands espaces forestiers appartenant au domaine public ${ }^{(3)} \mathrm{Or}$, quantité de terrains étajent situés en bordure des plans d'eau. Dans la foulée du démarrage touristique des années prócédant la deuxiême guerre mondiale, des citadins de plus en plus nombreux allaient occuper illégalement ces rives pour pratiquer des activités de chasse et de peche. La multiplication de ces squatters dans la région de Saint-Donat, entre autres, devaient accélérer l'action gouvernementale, ${ }^{(4)}$ 


\section{Les interventions gouvernementales}

a) 1938-1972: la *democratisation* de la résidence secondaire

C'est en 1938 que le MTF formula le premier décret qui posait les conditions et les exigences de la location des terres publiques pour fins de villégiature privée is' Le locataire devait signer un bail d'une durée maximale de 10 ans avec une possibilité de renouvellement. Comme it $n$ "y avait encore aucune politique établie sur le sujet, la pratique courante voulait qu'un minimum de 6 personnes environ suffise pour que le ministère permette l'ouverture d'un nouveau plan d'eau à la villégiature privée. Cette façon de procéder devait conduire à un développement anarchique de résidences secondaires dans l'espace à cause de l'absence presque totale de planification et de contrôle.

Entre 1938 et 1972, wplus de 20000 baux ont été consentis, lesquels visent en majorité des emplacements de villégiature familiale, groupés dans quelque 250 colonies riveraines de lacs ef de rivieres, et dont l'aménagement a favorisé l'économie régionale, tant à l'avantage des particuliers que des corps publicsw. ${ }^{(6)}$ Plusieurs de ces baux cependant se sont transformés en actes de vente sous la forme de lettres patentes. C'est à partir de 1958 que le gouvernement' commence à vendre des terrains jusque là loués à des villégiateurs. On a justifié cette nouvelle conduite par le fait qu'un grand nombre de locataires avaient érigé un ou des immeubles et apporté des améliorations à caractère permanent sur les lieux; ces derniers désiraient donc obtenir la propriété pleine et entière du terrain avant d'investir davantage.

Des décrets successifs (1958, 1959 et 1960) sont venus préciser le nombre maximum de ventes permises et les cantons désignés à cet effet. Entre 1958 et 1965 , plus de 3000 emplacements ont ainsi été vendus ${ }^{(7)}$ En 1965 et 1972, des textes de loi vont étendre le droit de vente. sans imposer de limites, à l'ensemble du Québec. Par cette loi, l'État exigeait néanmoins que si les conditions du bail locatif étaient respectées, la vente ne soit possible qu'après deux ans et qu'une réserve légale de trois chaines reste propriété publique. ${ }^{(8)}$ L'État a également concédé par acte de vente des terrains riverains ou non à des personnes ou groupes de personnes intéressés par des projets de lotissement d'habitats de loisir sans exiger l'examen de leur projet. Cette façon de faire ne fit qu'accentuer l'incohérence du développement. Cependant, avec la création en 1974 du Service de l'aménagement des terres (MTF). on exigea dorénavant le dépốt d'un plan d'aménagement du développement prévu afin de procéder à son évaluation selon des critères précis. Un décret fixant les prix et les conditions de la transaction était émis pour chaque projet soumis et accepté.

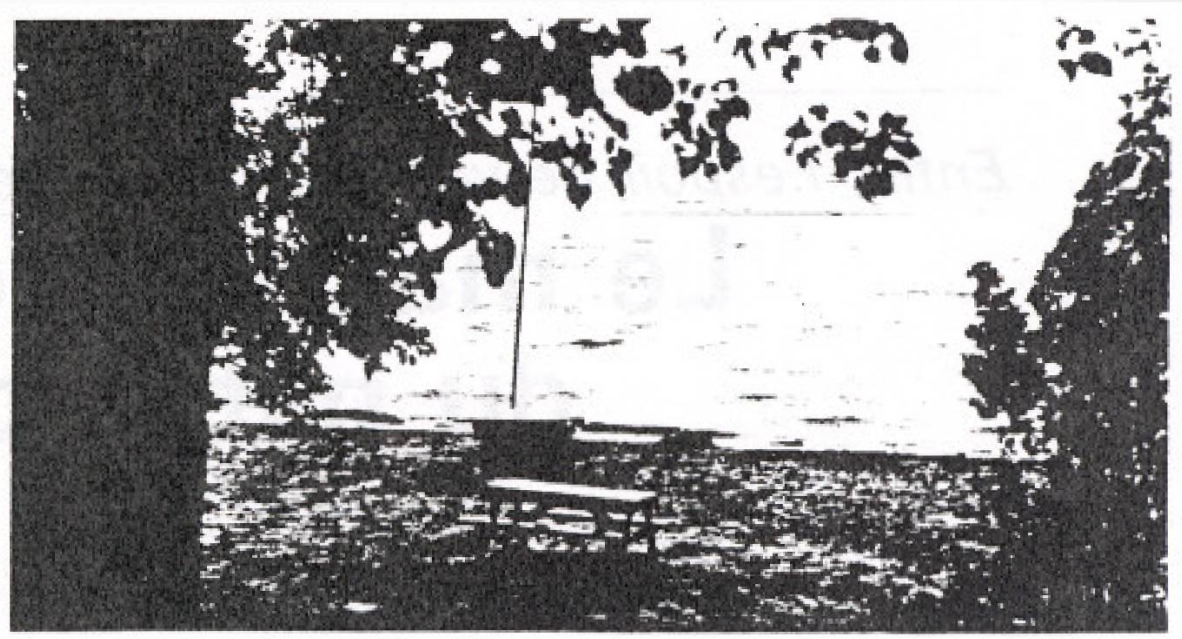

b) Les débuts d'une politique d'aménagement et ses effets

Suite à un moratoire d'un an, l'État effectua un changement important d'orientation avec l'énoncé en 1977 d'une politique d'accessibilité visant la préservation des terres. Cette politique atient compte du fair que le sol québécois est une richesse collective qui doit être gérée pour le bénéfice de la collectivité québécoise, et que conséquemment la collectivité doir donc y avoir accès. Pour les fins de villegiature, celte politique signifie que les lacs serant désormais aménagés de façon à prévoir, outre une partie pour des fins communautaires. une partie conservée à l'étal naturels. Cette nouvelle politique se voulait beaucoup plus restrictive afin d'éviter la spéculation foncière et préserver l'inaliénabilité du territoire. Pour arrêter la privatisation des rives, on proposait désormais des emplacements non riverains. De plus, des normes environnementales plus sévères étaient émises.

Toutefois, après quelques années, le gouvernement a constaté que son concept d'inaliénabilité des terres publiques, proposé comme principe essentiel à une meilleure gestion a long terme du domaine public, ne correspondait pas aux antentes de la clientèle. Le villégiateur québécois désire devenir proprietaire de son terrain plutôt que d'en demeurer le locataire. Ainsi, en 1982, la vente des terrains loués depuis 1977 est autorisée. ${ }^{110}$ Cependant, ce réajustement seul n'a pas augmenté le nom= bre de demandes de location et de ventes de terrains. Les lots offerts et disponibles sont trop éloignés des centres urbains et ne sont plus en bordure des plans d'eau: de plus, une situation économique difficile el l'effet pervers de normes environnementa. les qui augmentent les coúts d'installation ont contribué à freiner le développement de la villégiature privée.

\section{La fin de la résidence secondaire?}

Il apparait évident que, depuis 1938, le gouvernement québécois a joué un róle important dans le développement de la résidence secondaire; cette intervention a pro- bablement entrainé le secteur privé a profiter de ce marché en expansion. De même, les économies locale et régionale ont, jusqu'à un certain point, bénéficié de la venue de ces *nouveaux résidents* par le biais de la taxation municipale ou par les dépenses en biens et services.

Finalement, outre les facteurs que nous avons déjà énumérés, la baisse importante du marché de la résidence secondaire $=$ traditionnelle-"11n semble attribuable à des changements structurels au sein de la population. La clientele habituelle était le ménage d'äge moyen avec les enfants; or, on assiste à la fois à un vieillissement de la population et à l'augmentation des ménages sans enfants. Il faut aussi se rappeler que les années de grande prospérité économique sont terminées... Les MRC les plus concernées devront donc en tenir compte dans leur schéma d'aménagement. $f$

\section{Notes et rifterances}

(1) BUIES. Arthur, Au Portique des Laurantides. Une paroisse modeme, Quebac, Darvesu, 1891. p. 38

121 BLANCHARD, Raoul, Le Cantre du Canada. trançais, Montreal, Beauchemin, 1948, p. 524.

13) Depuis 1979, le ministere des Terres et Forless est devenu le ministere de l'Energie et des Res. sources.

(4) Sauf dans les regions où la villegiature est impor: tante, a proximite de la region metropolitaine notamment, il ne faut pas croire qu'it n'existe plus de squatters aupourd'hui.

[5] Superficie maximale autorisee de 5 acres $120000^{72}$ ou $217800 \mathrm{pi}^{2}$; : loyer annuel minimum a 15 * l'acre.

(6) Decret 541 11972), MTF

(7) Le prix ess fixé a au mons 250 s l'acre

(8) Bande de terrain de trois chalnes en protondeur. c'est-a dire 60 metres environ, reservet par la couronne le long des riveres et des lacs des Lau. rentides montrialases particulierement.

19) Politique annonce danș un communiqué émis en novembre 1977 .

190) Le loyer annuel est toxé da un minimum de 110 s pour 2,5 acres et le prix de vente a $1476 \leqslant$ pour la méme dimension. Précisons que l'augmentation s'est faite graduellemen par l'emission d'ordonnances enve 1956 et 1962 qui ajustaent les montants exiges.

(11) La residence secondaire ktraditionnelew, c'esta-dire celle gut etan accessible a une fore proportion de la population a revenus movens. 